\title{
Simon H./ Funk-Baker G. (2017), Deutsche Rechtssprache. Ein Stu- dien- und Arbeitsbuch mit Einführung in das deutsche Recht, 6., neubearbeitete Auflage, C.H. Beck u.a. München u.a., S. 334. ISBN 978-3-406-69256-7, 978-3-7190-3819-9, 978-3-7007-7105-0
}

Das Jurastudium zählt zu den beliebtesten Studiengängen in Deutschland. Zudem bieten die Universitäten vor dem Hintergrund der angestrebten Internationalisierung teilweise spezielle Studienrichtungen für Auslandsstudenten an, somit bedarf es dringend geeigneter Einführungen sowohl in das deutsche Recht, als auch in die deutsche Rechtssprache. Diesem Bedarf kommt u.a. ${ }^{1}$ seit der Erstauflage 1999 die nun in der 6. Auflage erschienene Publikation von Simon und Funk-Baker nach, welche 2017 erstmals unter einem neuen Titel herausgegeben wurde: Zuvor lautete der Titel Einführung in das deutsche Recht und die deutsche Rechtssprache. Die Titeländerung resultiert daraus, dass die Publikation Teil der Reihe Rechtssprache des Auslands ist und seit 2015 auch andere Werke dieser Reihe nach dem gleichen Muster betitelt werden, etwa Spanische Rechtssprache. Ein Studien- und Arbeitsbuch.

Verglichen mit den früheren Auflagen erweist sich die Struktur der neuesten Publikation seit der 4. Auflage aus dem Jahr 2009, was die Hauptkapitel anbelangt, als konstant und setzt sich aus 11 Hauptkapiteln zusammen. 2009 wurde es um das 10. Kapitel ergänzt, dessen Titel in der 5. Auflage zu Internationales Privatrecht, Europäisches Gemeinschaftsrecht und Europäische Menschenrechtskonvention modifiziert wurde. Im Vergleich zu der 5. Auflage aus dem Jahr 2013 enthält die neueste Ausgabe zusätzlich ein Unterkapitel zum Vertragsrecht - Geschäftsführung ohne Auftrag (S. 76), sowie ein Unterkapitel im Rahmen des Strafrechts - das Jugendstrafrecht (S. 145-146), welches eine klare Staffelung der Schuldfähigkeit bietet und angesichts der hohen Straffälligkeit unter Heranwachsenden auch von besonderer praktischer Relevanz ist. Eine unbestreitbare Stärke dieses Studienbuches ist die Tatsache, dass es solides Grundlagenwissen in den wichtigsten Gebieten des deutschen Rechts vermittelt. Dadurch kann sich der Benutzer einen Überblick über die grundlegenden Bereiche der deutschen Rechtsordnung verschaffen, aber auch Einblicke in die juristische Ausbildung, die Rechtsquellen sowie Fallbearbeitungen im Rahmen von Musterklausuren gewinnen. Gleichzeitig stellt jedes Kapitel eine abgeschlossene Einheit dar, so dass sich der Benutzer des Buches je nach Interesse auch lediglich mit ausgewählten Kapiteln auseinandersetzen kann (vgl. W. Wiesiołek 2005: 58). Ein Vorteil des Buches ist ebenso die Tatsache, dass es versucht, den Gesetzesänderungen nicht hinterherzuhinken, sondern dass tatsächlich in jedem

\footnotetext{
${ }^{1}$ Weitere zugängliche Publikationen, die z.T. ähnliche Adressaten anstreben sind beispielsweise I. Simonnæs (2015) oder S. Lippmann/ L. Scholz (2014).
} 
Kapitel Aktualisierungen, Modifizierungen, Ergänzungen, teilweise auch Verweise auf sich anbahnende Veränderungen vorgenommen werden.

Die im Klappentext angedeutete komplette Überarbeitung des Werkes bezieht sich somit sowohl auf die Form, als auch den Inhalt. Formal wurde die typographische Gestaltung modifiziert - dadurch, dass die Fallbeispiele nun durch Einrückungen von anderen Textteilen abgehoben, die Lösungshinweise durch Randschattierungen markiert, Aufgabenstellungen durch Fettdruck hervorgehoben und Unterkapitelüberschriften links ausgerichtet wurden, gewann das Buch eine größere Leserfreundlichkeit und Übersichtlichkeit. Nur vereinzelt ist das neue Layout nicht konsequent umgesetzt (keine Einrückung der Beispiele auf S. 67, im Inhaltsverzeichnis wird der Übungsteil nicht mehr in A. Rechtliche Aspekte und B. Sprachliche Aspekte eingeteilt, im Rahmen der methodischen Hinweise verweist man aber weiterhin noch auf die Einteilung in Übungen zum juristischen Inhalt (A) und zum Wortschatz (B) (S. XIV). Was den Inhalt anbelangt, so wird beim Vergleich der aktuellen Ausgabe mit der Auflage von 2013 offenbar, dass jedes Kapitel ausnahmslos ergänzt, aktualisiert oder modifiziert wurde. Im ersten Kapitel wird zusätzlich auf Einsatzmöglichkeiten von Juristen aus den Mitgliedsstaaten der Europäischen Union in Deutschland verwiesen (S. 14). Kapitel 2: Zugang zum Recht enthält neben einigen Aktualisierungen zusätzlich einen Absatz zur sog. ,verfassungskonformen Auslegung“ in der Rechtsprechung des BVerfG (S. 27). Vergleichsweise wenige Überarbeitungen findet man im Kapitel zum Verfassungsrecht, nur einzelne Sätze wurden darin ergänzt. Im vierten Kapitel zum Vertragsrecht wurden die Rechtsbeziehungen ohne Vertrag um Geschäftsführung ohne Auftrag ergänzt (S. 76), neben der Auflassung werden drei weitere Belastungen im Grundbuch thematisiert: Dienstbarkeiten, Grundschulden und Hypotheken (S. 78-79). Wichtig wäre es vielleicht noch auf die Deliktsfähigkeit im Zusammenhang mit den unerlaubten Handlungen einzugehen. Im Kapitel zum Familien- und Erbrecht wurden viele gesetzliche Neuerungen behandelt, etwa die Tatsache, dass die Güterstände in Eheverträgen neuerdings häufig individuell modifiziert werden (S. 94), die Schaffung der Möglichkeit der vertraulichen Geburt als Antwort auf die z.T. umstrittenen „Babyklappen“ (S. 95), weitere aktuelle Probleme im Kindschaftsrecht oder der Anstieg der Amtsvormundschaften angesichts des Zuzugs unbegleiteter minderjähriger Flüchtlinge (S. 97). Da das Studienbuch Ende Oktober 2017 erschienen ist, enthält es konsequenterweise noch keinen Verweis darauf, dass die eingetragene Lebenspartnerschaft nun nicht mehr begründet wird, da gleichgeschlechtlichen Paaren seit 1.10.2017 auch die Ehe als Rechtsinstitut zur Verfügung steht. Empfehlenswert in diesem Kapitel wäre die lediglich in Beispielform auf S. 89 zwingend geregelten Wirkungen der Ehe: die Pflicht zur ehelichen Lebensgemeinschaft, das Recht zur Berufstätigkeit und Unterhaltspflicht zusätzlich in dem gleich lautenden Unterkapitel zu erwähnen, sonst gewinnt man den Eindruck, dass zu Wirkungen der Ehe insbesondere das Namensrecht, Fragen der Haushaltsführung und steuerrechtliche Vorteile gehören (S. 91). Das sechste Kapitel wurde insbesondere durch Informationen zur sog. Stillen Gesellschaft (S. 113) bereichert. Das Kapitel zum Strafrecht wurde nicht nur um das Jugendstrafrecht ergänzt, es enthält auch Verweise auf eine sich anbahnende Reform 
der Tötungsdelikte (S. 141) sowie auf zahlreiche Änderungen im StGB (S. 141142). Außer dass bereits in der 5. Auflage ein Unterkapitel zum Umweltrecht im Rahmen des Verwaltungsrechts ergänzt wurde, ist Kapitel 8 in der 6. Ausgabe ausgenommen vereinzelter Datenaktualisierungen, etwa Höhe des Existenzminimums, der Grunderwerbsteuer, unverändert geblieben. Relativ wenige Veränderungen hat auch das Kapitel zum Verfahrensrecht erfahren: Die Autorinnen präzisieren hier den Anwendungsbereich außergerichtlicher Schlichtungsverfahren (S. 191) und thematisieren zusätzlich das Rechtsmittel der Sprungrevision (S. 194). Anpassungen an den heutigen aktuellen Rechtsstand in Form von kleinen Modifizierungen wurden auch im 10. Kapitel vorgenommen. Wünschenswert wäre es allerdings hier, die Frage des Anwendungsvorranges des Gemeinschaftsrechts ähnlich, wie das anderswo im Buch überzeugend veranschaulicht wird, auch an Fallbeispielen zu zeigen, stattdessen entsprechen knapp 60\% des Unterkapitels Vorrang des Gemeinschaftsrechts (S. 210-211) wortwörtlich dem letzten Absatz des Unterkapitels Europarecht und deutsches Recht (S. 58). Das letzte Kapitel mit den Musterklausuren enthält seit der 5. Auflage zusätzlich ein Beispiel für ein Zivilurteil. Die Tatsache, dass nicht alle Kapitel vergleichbar stark modifiziert wurden, korrespondiert natürlich damit, in welchem Ausmaß die jeweiligen Rechtsgebiete gesetzlich geändert wurden.

Was das Studienbuch von vergleichbaren Publikationen besonders abhebt, bilden die abwechslungsreichen jedem Kapitel folgenden Übungsteile. Dabei handelt es sich um sehr vielfältige Arbeitsanregungen, in die oft auch unterhaltsame Fallbeispiele eingebaut sind, was sich positiv auf das Lernen auswirkt. Bereits seit der Ausgabe von 2009 enthält jedes Kapitel zusätzlich im Rahmen der Übungen zu rechtlichen Aspekten zumindest eine rechtspolitische Frage, die auf eine kritische Stellungnahme zur Schaffung gänzlich neuer oder Änderung bestehender Regelungen zielt. Ferner wurde der Übungsteil zu juristischem Inhalt in der neuesten Ausgabe des Buches im Kapitel 4 um 5 weitere Aufgaben ergänzt und im Kapitel 7 um ein zusätzliches Beispiel. Es stellt sich somit heraus, dass die Publikation rein quantitativ gesehen, in ihrer vorherigen Ausgabe weit mehr Veränderungen und Ergänzungen erfahren hat als in der nun angekündigten komplett überarbeiteten 6. Ausgabe. 2013 wurde etwa im Kapitel 10 als einzigem Kapitel nicht nur eine zusätzliche Aufgabe zu rechtlichen Aspekten vorbereitet, sondern auch eine sprachliche Lückenergänzungsübung angeboten (S. 226).

Daraus mag eine gewisse Unbefriedigtheit resultieren. Infolge der Titelmodifizierung wurden nämlich bei den potentiellen Lesern gewisse Erwartungen an eine Schwerpunktverlagerung des Buches geweckt, die nicht gänzlich erfüllt werden, insbesondere wenn man bereits die früheren Auflagen kennt. Der Titel legt eine stärkere Fokussierung auf sprachliche Aspekte nahe, vergleicht man jedoch den jedem Kapitel nach rechtlichen Aspekten angeschlossenen Übungsteil zu sprachlichen Aspekten, so erweist sich dieser Teil des Buches als der einzige, der unverändert geblieben ist. Dabei wurde bereits bei Rezensionen früherer Auflagen festgestellt: „Jedoch kommen die sprachlichen Aspekte in diesem Buch zu kurz, es fehlt hier an einem breiteren Spektrum von Übungstypen, die zu einer tieferen sprachlichen Auseinandersetzung anregen könnten“ (P. Gergel 2014: 89). Gewiss handelt es 
sich auch bei einigen Übungen zu rechtlichen Aspekten um kommunikative Aufgaben, die folglich ebenso zu sprachlichen Produktionsaufgaben gerechnet werden können und somit mit Erfolg im Rahmen des Fachsprachenunterrichts zum Einsatz kommen können, die Trennung in sprachliche und rechtliche Aspekte, bei der im sprachlichen Übungsteil je zwei bis maximal vier Übungen dargeboten werden, im rechtlichen dagegen neun bis achtzehn lässt allerdings den Eindruck entstehen, dass der frühere Titel des Buches adäquater gewesen ist.

Einer Erwähnung bedarf noch die Tatsache, dass die Publikation dadurch gewinnen würde, wenn man sich an vereinzelten Textstellen noch stärker darum bemühen könnte, einige etwas verquaste, kompliziertere Formulierungen auszufeilen. Beispielweise findet man hier vereinzelt Formulierungen, die selbst von Juristen für Stilsünden erachtet werden, etwa die doppelte Verneinung (vgl. T. Walter 2017: 269-272). Da etwas „Verneintes [...] doppelt so schwer zu verstehen [ist] wie eine bejahende Aussage“" (T. Walter 2017: 115), wäre es beispielsweise überlegenswert, ob die Formulierung ,Soweit der Eigentümer nicht Immissionen (Lärm, Licht, Gerüche) als unwesentlich oder ortsüblich dulden muss, kann er grundsätzlich das Unterlassen der Störung verlangen“ (S. 79; Herv. F.K.) nicht gegen „Bei wesentlichen oder ortsunüblichen Immissionen kann der Eigentümer grundsätzlich das Unterlassen der Störung verlangen“" ersetzt werden könnte. Eine gewisse Unlogik liegt zwischen der Aufgabenstellung auf der S. 253 und dem Lösungsschlüssel (S. 273) vor. Die Übung beruht darauf, die falsche Verbform zu streichen, in dem Lösungsteil werden allerdings die passenden Verben angegeben, so dass etwa ein Autodidakt zu falschen Schlüssen geführt werden könnte.

Alles in allem stellt die hier besprochene Neuausgabe eine empfehlenswerte Lektüre zum Einstieg in das deutsche Recht dar. Der Benutzer hat die Möglichkeit, in die wichtigsten Rechtsgebiete eingeweiht zu werden, die anspruchsvolle Materie wird ihm anhand vieler Fallbeispiele, Definitionen und Übungen vermittelt (vgl. F. Księżyk 2018). Die Qualität und Quantität des Übungsteils hebt das Studienbuch von anderen vergleichbaren Publikationen ab.

\section{Bibliographie}

Gergel, P. (2014), Simon, Heike/Funk-Baker, Gisela: Einführung in das deutsche Recht und die deutsche Rechtssprache. München: Verlag C.H. Beck oHG, 5. Auflage 2013. ISBN 978-3-406-63658-5, (in:) „Slowakische Zeitschrift für Germanistik" 6/1, 87-89.

Księżyk, F. (2018/im Dr.), Wissens- und Wortschatzvermittlung im akademischen Fachfremdsprachenunterricht, (in:) I.-A. Busch-Lauer (ed.), Studien zu Fach, Sprache und Kultur. Bd. 6. Berlin.

Lippmann, S./ L. Scholz (2014), Das BGB für ausländische Studierende. Übungen zu Rechtssprache und Methodik. Heidelberg.

Simonnæs, I. (2015), Basiswissen deutsches Recht für Übersetzer. Mit Übersetzungsübungen und Verständnisfragen. Berlin. 
Walter, T. (2017), Kleine Stilkunde für Juristen. 3., gründlich überarbeitete Auflage. München.

Wiesiołek, W. (2005), Heike Simon, Gisela Funk-Baker, Einführung in das deutsche Recht und die deutsche Rechtssprache. 2. Auflage. C.H. Beck, München 2002, 282 s, (in:) „Przegląd Glottodydaktyczny” 2005/21, 57-61.

\section{Felicja KSIĘŻYK}

Uniwersytet Opolski / University of Opole

E-mail: ksiezykf@uni.opole.pl, 